Clinical Medicine

Poster

Abstract ID: 88

\title{
Lifestyles and non-communicable diseases status among primary health care staff in Kuantan, Pahang
}

\author{
Mohd Aznan Md Aris ${ }^{\mathrm{a}}$ | Tin Myo Han ${ }^{\mathrm{b}}$ | Nor Azwani Mohd Shukric | Fa'iza Abdullah \\ ${ }^{a}$ Department of Family Medicine, Kulliyyah of Medicine, International Islamic University Malaysia \\ ${ }^{b}$ Medical Statistic Unit, Kulliyyah of Dentistry, International Islamic University Malaysia \\ 'Department of Nutrition Sciences, Kulliyyah of Allied Health Sciences, International Islamic \\ University Malaysia
}

Introduction: Unhealthy lifestyle is one of the risks of non-communicable diseases (NCDs) in our population even among the healthcare provider. They should be as the role model for their NCDs patients in the clinics. Therefore, this study was to determine the lifestyle practice and lifestyle-related NCDs (LR-NCDs) status among primary health care staffs. Methods: A cross-sectional survey was done among 69 health-staff (20- physicians (PCPs), 16-nurses and 33-Para-Med) aged between 20-60 years from 3 selected governments primary health care clinics in Kuantan on April 2015. A pre-tested questionnaire is used to the collect history of LR-NCDs, smoking, exercise status and 24-hours- dietary recall. The BMI, systolic and diastolic blood pressure of the participants were also measured. The Nutritionist-Pro-(g) software was used to extract macronutrients (Carbohydrate, Protein and Total-Fat \%) and balance-diet (within recommended \%). Results: The most common LR-NCDs among health-staff were overweight (34.8\%) and followed by obesity $(24.6 \%)$, DM $(8.7 \%)$ and hypertension (3.2\%). The majority (95.7\%) were non-smokers and $69.6 \%$ did not exercise regularly. Diet-recall analysis showed that only 8.9\% (2-PCPs, 1-nurse \& 3-ParaMed) had balanced-diet. The status of the DM, hypertension and smoking and practicing balance-diet were not significantly different $(p>0.05)$ among the PCPs, registered-nurses and Para-Med except for the exercise. Most PCPs $(90 \%)$ have inadequate exercise compared to registered-nurses $(43.8 \%)$ and Para-Med $(69.7 \%)(p<0.05)$. Conclusions: There is a high prevalence of overweight and obesity, inadequate exercise and having an unbalance-diet were noticed among all levels of health-staff.

KEYWORDS: Lifestyle, NCDs, primary health care staff 